\title{
INDECOMPOSABLE DECOMPOSITIONS OF MODULAR STANDARD MODULES FOR TWO FAMILIES OF ASSOCIATION SCHEMES
}

\author{
AKIHIDE HANAKI AND OSAMU SHIMABUKURO
}

\begin{abstract}
We investigate the indecomposable decomposition of the modular standard modules of two families of association schemes of finite order. First, we show that, for each prime number $p$, the standard module over a field $F$ of characteristic $p$ of a residually thin scheme $S$ of $p$-power order is an indecomposable $F S$-module. Second, we describe the indecomposable decomposition of the standard module over a field of positive characteristic of a wreath product of finitely may association schemes of rank 2 .
\end{abstract}

\section{INTRODUCTION}

It is easy to see that algebraically isomorphic association schemes have isomorphic standard modules over algebraically closed fields of characteristic 0 . In [3], the first author considered modular adjacency algebras and standard modules of cyclotomic association schemes, and gave direct sum decompositions of the standard modules. He determined indecomposable decompositions only for the case where the representation types were finite, or tame and the dimensions of algebras were small. In general, it is hard to describe indecomposability of a given module, especially if the representation type of the algebra is wild. For representation types, see [6], for example.

In this article, we provide indecomposable decompositions of modular standard modules for two families of association schemes. Let $F$ be a field of positive characteristic $p$. In Section 4, we consider residually thin schemes of $p$-power order (called $p$-schemes in [8]). In this case, the standard modules are indecomposable (Theorem 4.2). To prove this, we consider multiple wreath products of thin schemes given by the cyclic groups of order $p$ and show that their standard modules are indecomposable. Since residually thin schemes are fissions of such wreath products, we see that their standard modules are indecomposable. In Section 5, we consider multiple wreath products of schemes of rank 2. This is one of the simplest examples of association schemes but the structure of their standard module is not always easy to describe. The adjacency algebra is isomorphic to

$$
F \oplus \cdots \oplus F \oplus F\left[u_{1}, \ldots, u_{n}\right] /\left(u_{i} u_{j} \mid 1 \leq i, j \leq n\right) .
$$

Therefore the representation type of the algebra is finite if $n=0,1$, tame if $n=2$, and wild if $n \geq 3$. We completely determine indecomposable decompositions of

2010 Mathematics Subject Classification. Primary (05E30); Secondary (16G10).

Key words and phrases. association scheme; wreath product; modular adjacency algebra; modular standard module; indecomposable decomposition. 
standard modules for them (Theorem 5.1). This is the first result on indecomposable decompositions for wild adjacency algebras of association schemes.

In Section 2, we give the definition of an association scheme. In Section 3, we define an algebra $V$ and its subalgebra $W$ and show that $V$ is indecomposable as a $W$-module (Proposition 3.3). This is a key result in this article.

Throughout this article, we denote by $I_{n}$ the identity matrix of degree $n$, and by $J_{n}$ the $n \times n$ matrix all of whose entries are one.

\section{Association schemes}

In this section, we will give necessary definitions for association schemes and their representations. For details, see $[1,8]$.

Let $X$ be a finite set, and let $S$ be a collection of non-empty subsets of $X \times X$. We call $(X, S)$ an association scheme or a scheme if the following conditions hold:

(1) $X \times X=\bigcup_{s \in S} s$ and $s \cap s^{\prime}=\emptyset$ for $s \neq s^{\prime}, s, s^{\prime} \in S$,

(2) $1_{X}=\{(x, x) \mid x \in X\} \in S$,

(3) $s^{*}=\{(y, x) \mid(x, y) \in s\} \in S$ for $s \in S$, and

(4) for $s, t, u \in S$, there is an integer $p_{s t}^{u}$ such that $\sharp\{z \in X \mid(x, z) \in s,(z, y) \in$ $t\}=p_{s t}^{u}$ whenever $(x, y) \in u$.

In this case, we also say that $S$ is an association scheme. The number $p_{s s^{*}}^{1_{X}}$ is called the valency of $s \in S$ and denoted by $n_{s}$. We call the number $|X|$ the order of the association scheme $(X, S)$. We denote by $M_{X}(R)$ the full matrix algebra over a commutative ring $R$ with unity, where both rows and columns of whose matrices are indexed by the set $X$. For $s \subset X \times X$, we define the adjacency matrix $A_{s} \in M_{X}(\mathbb{Z})$ by $\left(A_{s}\right)_{x y}=1$ if $(x, y) \in s$ and $\left(A_{s}\right)_{x y}=0$ otherwise. By definition, $\mathbb{Z} S=\bigoplus_{s \in S} \mathbb{Z} A_{s}$ is a subalgebra of $M_{X}(\mathbb{Z})$. Let $R$ be a commutative ring with unity. We define an $R$-subalgebra $R S=R \otimes_{\mathbb{Z}} \mathbb{Z} S$ of $M_{X}(R)$ and call it the adjacency algebra of $(X, S)$ over $R$. We also write $R \mathfrak{X}$ for the adjacency algebra of $\mathfrak{X}=(X, S)$. A representation of $(X, S)$ over $R$ is an $R$-algebra homomorphism from $R S$ to $M_{n}(R)$ for some degree $n$. Since $R S$ is defined as a subalgebra of $M_{X}(R)$, the inclusion map is a representation and we call it the standard representation of $(X, S)$ over $R$. The corresponding $R S$-module is called the standard module of $(X, S)$ over $R$. The standard module has a natural basis $X$, so we denote it by $R X$.

A subset $T$ of $S$ is said to be closed if $p_{s t}^{u}=0$ for all $s, t \in T$ and $u \notin T$. A closed subset defines subschemes and the factor scheme. An association scheme $(X, S)$ is said to be thin if $n_{s}=1$ for all $s \in S$. A thin association schemes are obtained by a regular permutation representation of a finite group. Thus a thin scheme is essentially a finite group. The thin residue $\mathbf{O}^{\theta}(S)$ is the smallest closed subset of $S$ such that the factor scheme $S / / \mathbf{O}^{\theta}(S)$ is thin. Define $\left(\mathbf{O}^{\theta}\right)^{n}(S)$ by $\mathbf{O}^{\theta}\left(\left(\mathbf{O}^{\theta}\right)^{n-1}(S)\right)$ inductively. An association scheme $(X, S)$ is said to be residually thin if $\left(\mathbf{O}^{\theta}\right)^{n}(S)=1$ for some $n$. For details, see [8].

Let $\mathfrak{X}=(X, S)$ and $\mathfrak{Y}=(X, T)$ be association schemes on a common underlying set $X$. When every $t \in T$ is a union of some subset of $S$, we say that $\mathfrak{Y}$ is a fusion of $\mathfrak{X}$ and $\mathfrak{X}$ is a fission of $\mathfrak{Y}$. In this case, the adjacency algebra $R T$ is a subalgebra of $R S$.

Let $\mathfrak{X}=(X, S)$ and $\mathfrak{Y}=(Y, T)$ be association schemes with adjacency matrices $\left\{A_{i}\right\}_{i=0}^{d}$ and $\left\{A_{i}^{\prime}\right\}_{i=0}^{f}$, respectively. We suppose that $A_{0}$ and $A_{0}^{\prime}$ are identity 
matrices. For $s \in S$ and $t \in T$, we set

$$
s \times t=\left\{\left((x, y),\left(x^{\prime}, y^{\prime}\right)\right) \mid\left(x, x^{\prime}\right) \in s,\left(y, y^{\prime}\right) \in t\right\} \subset(X \times Y) \times(X \times Y)
$$

and

$$
S \times T=\{s \times t \mid s \in S, t \in T\} .
$$

Then $(X \times Y, S \times T)$ is an association scheme, called the direct product of $\mathfrak{X}$ and $\mathfrak{Y}$, and denoted by $\mathfrak{X} \times \mathfrak{Y}$. The adjacency matrices of $\mathfrak{X} \times \mathfrak{Y}$ are $(d+1)(f+1)$ matrices:

For $s \in S$, we put

$$
A_{0} \otimes A_{0}^{\prime}, \ldots, A_{d} \otimes A_{f}^{\prime}
$$

$$
\tilde{s}=\left\{\left((x, y),\left(x^{\prime}, y\right)\right) \mid\left(x, x^{\prime}\right) \in s, y \in Y\right\} \subset(X \times Y) \times(X \times Y) .
$$

For $t \in T$, we put

$$
\tilde{t}=\left\{\left((x, y),\left(x^{\prime}, y^{\prime}\right)\right) \mid x, x^{\prime} \in X,\left(y, y^{\prime}\right) \in t\right\} \subset(X \times Y) \times(X \times Y) .
$$

Also we put

$$
S\left\{T=\{\tilde{s} \mid s \in S\} \cup\left\{\tilde{t} \mid t \in T \backslash\left\{1_{Y}\right\}\right\} .\right.
$$

Then $(X \times Y, S \imath T)$ is an association scheme, called the wreath product of $\mathfrak{X}$ and $\mathfrak{Y}$, and denoted by $\mathfrak{X}<\mathfrak{Y}$. The adjacency matrices of $\mathfrak{X}<\mathfrak{Y}$ are $d+f+1$ matrices:

$$
A_{0} \otimes I_{|Y|}, \ldots, A_{d} \otimes I_{|Y|}, J_{|X|} \otimes A_{1}^{\prime}, \ldots, J_{|X|} \otimes A_{f}^{\prime} .
$$

For irreducible representations of wreath products, see [4].

It is clear that the wreath product $\mathfrak{X}<\mathfrak{Y}$ is a fusion scheme of $\mathfrak{X} \times \mathfrak{Y}$. This means that $R(S \succ T)$ is a subalgebra of $R(S \times T)$.

Let $\mathfrak{X}=(X, S)$ be an association scheme. The cardinality of $S$ is called the rank of $\mathfrak{X}$. For any finite set $X$ with $|X| \geq 2$, there is a unique association scheme on $X$ of rank 2 , namely it is $\left(X,\left\{1_{X},(X \times X) \backslash 1_{X}\right\}\right)$.

Let $\mathfrak{X}=(X, S)$ be an association scheme with a closed subset $T$. A subscheme $\mathfrak{Y}_{x}=(X, S)_{x T}$ (see $[8, \S 1.5]$ for definition) depends on $x \in X$. Subschemes are algebraically isomorphic but not necessarily isomorphic. We will give an easy lemma without a proof.

Lemma 2.1. Let $\mathfrak{X}=(X, S)$ be an association scheme with a closed subset $T$. Suppose that all subschemes $\mathfrak{Y}_{x}(x \in X)$ of $\mathfrak{X}$ defined by $T$ are isomorphic. Then $\mathfrak{X}$ is a fission scheme of the wreath product $\mathfrak{Y}_{x} \prec(S / / T)$.

\section{IndECOMPOSABILITY OF A MODULE}

In this section, for $n_{i} \geq 2(i=1, \ldots, r)$, we define an algebra $V=V\left(n_{1} \ldots, n_{r}\right)$ and a subalgebra $W=W\left(n_{1}, \ldots, n_{r}\right)$ and prove that $V$ is indecomposable as a $W$-module in Proposition 3.3 .

Let $F$ be a field and $n_{1}, \ldots, n_{r}$ be integers which are greater than or equal to 2 . We define a finite dimensional $F$-algebra $V$ by

$$
V=V\left(n_{1}, \ldots, n_{r}\right)=F\left[t_{1}, \ldots, t_{r}\right] /\left(t_{1}^{n_{1}}, \ldots, t_{r}^{n_{r}}\right) .
$$

We write $t_{i}$ in the factor ring $V$ by the same letter $t_{i}$. The set

$$
\mathbf{B}=\left\{t_{1}^{e_{1}} \ldots t_{r}^{e_{r}} \mid 0 \leq e_{i}<n_{i} \quad(1 \leq i \leq r)\right\}
$$


is a basis of $V$. We use the lexicographical order in sequences of powers for the basis and write a base $\boldsymbol{t}^{e}=t_{1}^{e_{1}} \ldots t_{r}^{e_{r}}$ for $e=\left(e_{1}, \ldots, e_{r}\right)$. Let us put $V_{>e}=\bigoplus_{f>e} F \boldsymbol{t}^{f}$. For any element $\alpha$ of $V$, we can write uniquely as

$$
\alpha=c_{e} \boldsymbol{t}^{e}+\beta, \quad\left(c_{e} \in F \backslash\{0\}, \beta \in V_{>e}\right) .
$$

We consider a subalgebra $W=W\left(n_{1}, \ldots, n_{r}\right)$ of $V$ with basis

$$
\mathbf{B}^{\prime}=\{1\} \cup\left(\bigcup_{i=1}^{r}\left\{t_{1}^{n_{1}-1} \ldots t_{i-1}^{n_{i-1}-1} t_{i}^{e_{i}} \mid 1 \leq e_{i} \leq n_{i}-1\right\}\right) .
$$

We can consider $V$ as a right $W$-module.

By considering products of elements in $\mathbf{B}^{\prime}$, easily we have the structure of $W$, that is

$$
W=F\left[u_{0}, u_{1}, \ldots, u_{\ell}\right] / \mathcal{I},
$$

where $\ell=\sum_{i=2}^{r}\left(n_{i}-1\right)$ and $\mathcal{I}$ is the ideal generated by

$$
\left\{u_{0}^{n_{1}}\right\} \cup\left\{u_{i}^{2} \mid 1 \leq i \leq \ell\right\} \cup\left\{u_{i} u_{j} \mid 0 \leq i<j \leq \ell\right\} .
$$

We remark that the algebra $W$ has wild representation type when $\ell \geq 2$ (see [6], for example), and representation theory is difficult in this case.

We show that the endomorphism algebra $\operatorname{End}_{W}(V)$ is a local algebra so that we prove $V_{W}$ is indecomposable.

Lemma 3.1. For $\varphi \in \operatorname{End}_{W}(V)$ and $0 \neq \alpha \in \operatorname{Ker} \varphi$, put $\alpha=c_{e} \boldsymbol{t}^{e}+\beta\left(c_{e} \in\right.$ $\left.F \backslash\{0\}, \beta \in V_{>e}\right)$, we suppose that $e<\left(n_{1}-1, \ldots, n_{r}-1\right)$. Then there exist a sequence $f$ and $0 \neq \alpha^{\prime}=c_{f} \boldsymbol{t}^{f}+\gamma\left(c_{f} \in F \backslash\{0\}, \gamma \in V_{>f}\right)$ such that $f>e$ and $\alpha^{\prime} \in \operatorname{Ker} \varphi$.

Proof. We put $e=\left(n_{1}-1, \ldots, n_{i-1}-1, e_{i}, \ldots, e_{r}\right)$, where $e_{i}<n_{i}-1$. We remark that $t_{1}^{n_{1}-1} \ldots t_{i-1}^{n_{i-1}-1} t_{i}^{e_{i}} \in \mathbf{B}^{\prime}$ is a common factor of each term of $\beta$. Since $\alpha \in \operatorname{Ker} \varphi$,

$$
0=\varphi(\alpha)=\varphi\left(c_{e} t_{i+1}^{e_{i+1}} \ldots t_{r}^{e_{r}}+\beta^{\prime}\right) t_{1}^{n_{1}-1} \ldots t_{i-1}^{n_{i-1}-1} t_{i}^{e_{i}} .
$$

Multiplying both sides of this equation by $t_{i}^{n_{i}-e_{i}-1}$, since $t_{1}^{n_{1}-1} \ldots t_{i-1}^{n_{i-1}-1} t_{i}^{n_{i}-1} \in$ $\mathbf{B}^{\prime}$, we have

$$
0=\varphi\left(c_{e} t_{i+1}^{e_{i+1}} \ldots t_{r}^{e_{r}}+\beta^{\prime}\right) t_{1}^{n_{1}-1} \ldots t_{i-1}^{n_{i-1}-1} t_{i}^{n_{i}-1}=\varphi\left(c_{e} \boldsymbol{t}^{e} t_{i}^{n_{i}-e_{i}-1}+\beta t_{i}^{n_{i}-e_{i}-1}\right) .
$$

If we put $\alpha^{\prime}=c_{e} \boldsymbol{t}^{e} t_{i}^{n_{i}-e_{i}-1}+\beta t_{i}^{n_{i}-e_{i}-1}$, then the conditions are satisfied.

Lemma 3.2. For $\varphi \in \operatorname{End}_{W}(V), \varphi$ is an isomorphism if and only if $\varphi\left(\boldsymbol{t}^{\left(n_{1}-1, \ldots, n_{r}-1\right)}\right) \neq$ 0 .

Proof. If $\varphi$ is an isomorphism, then it is clear that $\varphi\left(\boldsymbol{t}^{\left(n_{1}-1, \ldots, n_{r}-1\right)}\right) \neq 0$. We assume that $\varphi$ is not an isomorphism. This means that $\operatorname{Ker} \varphi \neq 0$. By repeatedly using Lemma 3.1, we can conclude that $\boldsymbol{t}^{\left(n_{1}-1, \ldots, n_{r}-1\right)} \in \operatorname{Ker} \varphi$.

Now we can prove the main result in this section.

Proposition 3.3. The endomorphism algebra $\operatorname{End}_{W}(V)$ is a local algebra. Namely, $V$ is indecomposable as a right $W$-module.

Proof. If $\varphi$ is a non-isomorphism and $\psi \in \operatorname{End}_{W}(V)$, then clearly $\varphi \psi$ and $\psi \varphi$ are non-isomorphisms. Suppose that both $\varphi$ and $\psi$ are non-isomorphisms. Then, by Lemma 3.2, $\varphi+\psi$ is not an isomorphism. Hence the set of all non-isomorphisms in $\operatorname{End}_{W}(V)$ is an ideal of $\operatorname{End}_{W}(V)$, and thus $\operatorname{End}_{W}(V)$ is local by $[5, \mathrm{I}, \S 5]$. 
We will give a matrix form of Proposition 3.3. We consider a regular representation of $V$. Put $N_{n}$ the $n \times n$ matrix of the form

$$
N_{n}=\left(\begin{array}{ccccc}
0 & 1 & & & \\
& 0 & 1 & & \\
& & \ddots & \ddots & \\
& & & \ddots & 1 \\
& & & & 0
\end{array}\right) .
$$

Then $\Phi: t_{1}^{e_{1}} \ldots t_{r}^{e_{r}} \mapsto N_{n_{1}}^{e_{1}} \otimes \cdots \otimes N_{n_{r}}^{e_{r}}$ is a regular representation of $V$.

Proposition 3.4. The restriction $\Phi$ to $W$ is an indecomposable representation of $W$.

\section{Residually thin SChemes OF PRIME POWER ORDER}

Let $F$ be a field of characteristic $p$, and let $(X, S)$ be a residually thin association scheme of $p$-power order, a $p$-scheme in [8]. In this section, we will show that the standard module $F X$ is an indecomposable $F S$-module. Thus the indecomposable decomposition of the standard module $F X$ is determined for this case.

We need one lemma.

Lemma 4.1. Let $F$ be a field of characteristic $p$, and let $\mathfrak{C}_{p}$ be the association scheme given by the regular permutation representation of the cyclic group order $p$. Then the standard module of the wreath product $\mathfrak{C}_{p} 2 \cdots \cdot \mathfrak{C}_{p}$ over $F$ is indecomposable.

Proof. We can write $\mathfrak{C}_{p}=\left(C_{p}, C_{p}\right)$, where $C_{p}$ is the cyclic group of order $p$. We know that $F \mathfrak{C}_{p} \cong F[t] /\left(t^{p}\right)$ and the all-one matrix $J_{p}$ corresponds to $t^{p-1}$. The standard module of $\mathfrak{C}_{p} 2 \cdots \gamma \mathfrak{C}_{p}(r$-times $)$ is $F\left(C_{p} \times \cdots \times C_{p}\right)$. The module $F\left(C_{p} \times\right.$ $\left.\cdots \times C_{p}\right)$ has a natural algebra structure and it is easy to see that

$$
\begin{aligned}
F\left(C_{p} \times \cdots \times C_{p}\right) & \cong F C_{p} \otimes \cdots \otimes F C_{p} \\
& \cong F[t] /\left(t^{p}\right) \otimes \cdots \otimes F[t] /\left(t^{p}\right) \\
& \cong F\left[t_{1}, \ldots, t_{r}\right] /\left(t_{1}^{p}, \ldots, t_{r}^{p}\right) \\
& =V(p, \ldots, p) .
\end{aligned}
$$

Now the adjacency algebra $F\left(\mathfrak{C}_{p} \prec \cdots \prec \mathfrak{C}_{p}\right)$ is a subalgebra of $F\left(C_{p} \times \cdots \times C_{p}\right)$, and since $J_{p}$ corresponds $t^{p-1}$, we have $F\left(\mathfrak{C}_{p}\left\langle\cdots \cdot \mathfrak{C}_{p}\right) \cong W(p, \ldots, p)\right.$. Thus the standard module is indecomposable by Proposition 3.3 .

Now we show one of the main results in this article.

Theorem 4.2. Let $F$ be a field of characteristic $p$, and let $(X, S)$ be a residually thin association scheme of p-power order. Then the standard module $F X$ is an indecomposable FS-module.

Proof. By definition, there is a series of closed subsets

$$
S=S_{0} \supset S_{1} \supset \cdots \supset S_{r}=1
$$

such that $S_{i-1} / / S_{i} \cong C_{p}$ for $i=1, \ldots, r$. Therefore, $(X, S)$ is a fission scheme of $\left.\mathfrak{C}_{p} \imath \cdots\right\urcorner \mathfrak{C}_{p}$ by Lemma 2.1 and the adjacency algebra $\left.F\left(\mathfrak{C}_{p} \imath \cdots\right\urcorner \mathfrak{C}_{p}\right)$ is a subalgebra of $F S$. We can assume that the standard modules $F X$ are common. The standard module is indecomposable as an $F\left(\mathfrak{C}_{p} \prec \cdots \prec \mathfrak{C}_{p}\right)$-module by Lemma 4.1, and thus it is indecomposable as an $F S$-module. 
We give an easy application as a corollary to Theorem 4.2, though it is not so hard to prove it by combinatorial argument [8, Corollary 2.4.7]. An association scheme $(X, S)$ is said to be $p^{\prime}$-valenced if $p \nmid n_{s}$ for all $s \in S$.

Corollary 4.3. Let $(X, S)$ be a $p^{\prime}$-valenced residually thin association scheme of p-power order. Then $(X, S)$ is thin.

Proof. We fix $x \in X$. The map $f: F S \rightarrow F X, f\left(A_{s}\right)=x A_{s}$ is an $F S$-module monomorphism. Since $(X, S)$ is $p^{\prime}$-valenced, $F S$ is a symmetric algebra by $[2$, Corollary 4.3] and thus $F S$ is an injective $F S$-module. Therefore the map $f$ splits, namely $F S$ is isomorphic to a direct summand of $F X$. However, by Theorem 4.2, $F X$ is indecomposable. This is possible only if $|S|=|X|$, namely $(X, S)$ is thin.

\section{WREATH PRODUCT OF SCHEMES OF RANK 2}

Let $X_{1}, \ldots, X_{r}$ be finite sets. For each element $i \in\{1, \ldots, r\}$, let $S_{i}$ be an association scheme of order $q_{i}(\geq 2)$ and rank 2 on $X_{i}$. Define $X=X_{1} \times \cdots \times X_{r}$ and $S=S_{1} \imath \cdots 2 S_{r}$. Then $S$ is an association scheme on $X$. Association schemes of this type have been characterized in [7, Theorem A]. The adjacency matrices are described as follows

$$
A_{i}=J_{q_{1}} \otimes \cdots \otimes J_{q_{i-1}} \otimes\left(J_{q_{i}}-I_{q_{i}}\right) \otimes I_{q_{i+1}} \otimes \cdots \otimes I_{q_{r}} \quad(i=0,1, \ldots, r) .
$$

We replace the basis $\left\{A_{i} \mid i=0,1, \ldots, r\right\}$ of the adjacency algebra with $\left\{B_{i} \mid i=\right.$ $0,1, \ldots, r\}$ where

$$
B_{i}=\sum_{j=0}^{i} A_{j}=J_{q_{1}} \otimes \cdots \otimes J_{q_{i}} \otimes I_{q_{i+1}} \otimes \cdots \otimes I_{q_{r}} \quad(i=0,1, \ldots, r) .
$$

Let $F$ be a field of characteristic $p$. The adjacency algebra is a subalgebra of $M_{X_{1}}(F) \otimes \cdots \otimes M_{X_{r}}(F)$ with basis $\left\{B_{i} \mid i=0,1, \ldots, r\right\}$ and the standard module is $F\left(X_{1} \times \cdots \times X_{r}\right) \cong F X_{1} \otimes \cdots \otimes F X_{r}$.

The space $F X_{i}$ has a natural basis $X_{i}=\left\{x_{1}, \ldots, x_{q_{i}}\right\}$. For each $i \in\{1, \ldots, r\}$, we replace this basis with $\left\{y_{1}, \ldots, y_{q_{i}}\right\}$ where

$$
y_{1}=x_{1}, \quad y_{2}=\sum_{j=1}^{q_{i}} x_{j}, \quad y_{k}=x_{k}-x_{1} \quad\left(k=3,4, \ldots, q_{i}\right),
$$

if $p \mid q_{i}$, and

$$
y_{1}=\sum_{j=1}^{q_{i}} x_{j}, \quad y_{k}=x_{k}-x_{1} \quad\left(k=2,3, \cdots, q_{i}\right) .
$$

if $p \nmid q_{i}$. The representing matrix of $J_{q_{i}}$ with respect to the basis $\left\{y_{1}, \ldots, y_{q_{i}}\right\}$ is

$$
\left(\begin{array}{cccc}
0 & 1 & \cdots & 0 \\
0 & 0 & \cdots & 0 \\
& \cdots & \cdots & \\
0 & \cdots & \cdots & 0
\end{array}\right) \text { if } p \mid q_{i} \text {, and }\left(\begin{array}{cccc}
q_{i} & 0 & \cdots & 0 \\
0 & 0 & \cdots & 0 \\
& \cdots & \cdots & \\
0 & \cdots & \cdots & 0
\end{array}\right) \text { if } p \nmid q_{i} \text {. }
$$

We set

- $\Delta=\left\{i|1 \leq i \leq r, p| q_{i}\right\}$,

- $T_{i}=\{1,2\}$ if $i \in \Delta$ and $T_{i}=\{1\}$ if $i \notin \Delta$,

- $U_{i}=\bigoplus_{j \in T_{i}} F y_{j}$, and

- $\delta(k)=|\Delta \cap\{1, \ldots, k\}|$. 
Now we show the main result in this section.

Theorem 5.1. Let $\left(X_{i}, S_{i}\right), i=1,2, \ldots, r$, be association schemes of rank 2 . We have an indecomposable decomposition of the standard module of $(X, S)=$ $\left(X_{1} \times \cdots \times X_{r}, S_{1} \imath \cdots \imath S_{r}\right)$ :

$$
F X=F X_{1} \otimes \cdots \otimes F X_{r}=\bigoplus_{i=0}^{r} \bigoplus U_{1} \otimes \cdots \otimes U_{i} \otimes F y_{\ell_{i+1}} \otimes \cdots \otimes F y_{\ell_{r}},
$$

where the second direct sum runs over all $\left(\ell_{i+1}, \ldots, \ell_{r}\right)$ such that $\ell_{i+1} \notin T_{i+1}$ and $1 \leq \ell_{k} \leq q_{k}(k=i+2, \ldots, r)$. Moreover, the indecomposable direct summands $U_{1} \otimes \cdots \otimes U_{i} \otimes F y_{\ell_{i+1}} \otimes \cdots \otimes F y_{\ell_{r}}$ and $U_{1} \otimes \cdots \otimes U_{i^{\prime}} \otimes F y_{\ell_{i^{\prime}+1}^{\prime}} \otimes \cdots \otimes F y_{\ell_{r}^{\prime}}$ are isomorphic as FS-modules if and only if $i=i^{\prime}$.

Proof. It is easy to see that the sum is direct and every direct summand is an $F S$-submodule of $F X$.

We consider a direct summand $U=U_{1} \otimes \cdots \otimes U_{i} \otimes F y_{\ell_{i+1}} \otimes \cdots \otimes F y_{\ell_{r}}$. We set

$$
M_{k}=\left(\begin{array}{ll}
0 & 1 \\
0 & 0
\end{array}\right) \text { if } k \in \Delta, \text { and } M_{k}=\left(q_{k}\right) \text { if } k \notin \Delta .
$$

Then the action of $B_{j}$ on $U$ is

$$
M_{1} \otimes \cdots \otimes M_{j} \otimes I \otimes \cdots \otimes I
$$

for $0 \leq j \leq i$, where $I$ is the identity matrix of degree 1 or 2 , and 0 for $j>i$. By the action of $\left\{B_{i}\right\}$ on the summands, we can characterize isomorphic direct summands. We need to show that $U$ is indecomposable. We consider a subalgebra $W$ of $F S$ generated by $\left\{B_{j} \mid 1 \leq j \leq i, j \in \Delta\right\}$. We can see that $W \cong W(2, \ldots, 2)(\delta(i)$ times), defined in section 3 , and the action of $W$ on $U$ is just the representation in Proposition 3.4 up to non-zero scalar factors. Thus $F X$ is an indecomposable $W$-module, and so is an indecomposable $F S$-module.

We set

$$
V_{i}=U_{1} \otimes \cdots \otimes U_{i} \otimes F y_{\ell_{i+1}} \otimes \cdots \otimes F y_{\ell_{r}}
$$

where $\ell_{i+1} \notin T_{i+1}$. The dimension of $V_{i}$ is $2^{\delta(i)}$ and $V_{i}$ appears $\left(q_{i+1}-\mu\right) \prod_{k=i+2}^{r} q_{k}$ times in $F X$, where $\mu=2$ if $p \mid q_{i+1}$ and $\mu=1$ otherwise.

If $p \nmid q_{j}$ for $1 \leq j \leq m-1$ and $p \mid q_{m}$, then $\left\{V_{0}, \cdots, V_{m}\right\}$ is the complete set of isomorphism classes of simple $F S$-modules, $V_{k}$ is not a simple $F S$-module if $k>m$, and all composition factors of $V_{k}$ are $V_{m}$ if $k>m$.

The structure of the adjacency algebra $F S$ is

$$
F S \cong \underbrace{F \oplus \cdots \oplus F}_{m \text {-times }} \oplus F\left[u_{1}, \ldots, u_{r-m}\right] /\left(u_{i} u_{j} \mid 1 \leq i, j \leq r-m\right) .
$$

Therefore the representation type of $F S$ is finite if $0 \leq r-m \leq 1$, tame if $r-m=2$, and wild if $r-m \geq 3$.

\section{ACKnowledgment}

The authors would like to thank the anonymous referees for their helpful comments. This work was supported by JSPS KAKENHI Grant Number JP25400011. 


\section{REFERENCES}

1. E. Bannai and T. Ito, Algebraic combinatorics. I, The Benjamin/Cummings Publishing Co. Inc., Menlo Park, CA, 1984.

2. A. Hanaki, Locality of a modular adjacency algebra of an association scheme of prime power order, Arch. Math. (Basel) 79 (2002), no. 3, 167-170.

3. __ Modular adjacency algebras, standard representations, and p-ranks of cyclotomic association schemes, J. Algebraic Combin. 44 (2016), no. 3, 587-602.

4. A. Hanaki and K. Hirotsuka, Irreducible representations of wreath products of association schemes, J. Algebraic Combin. 18 (2003), no. 1, 47-52.

5. H. Nagao and Y. Tsushima, Representations of finite groups, Academic Press Inc., Boston, MA, 1989.

6. C. M. Ringel, The representation type of local algebras, Proceedings of the International Conference on Representations of Algebras (Carleton Univ., Ottawa, Ont., 1974), Paper No. 22, Carleton Univ., Ottawa, Ont., 1974, pp. 24 pp. Carleton Math. Lecture Notes, No. 9.

7. R. Tanaka and P.-H. Zieschang, On a class of wreath products of hypergroups and association schemes, J. Algebraic Combin. 37 (2013), no. 4, 601-619.

8. P.-H. Zieschang, An algebraic approach to association schemes, Lecture Notes in Mathematics, vol. 1628, Springer-Verlag, Berlin, 1996.

(Akihide Hanaki) Faculty of Science, Shinshu University, Matsumoto, 390-8621, Japan Email address: hanaki@shinshu-u.ac.jp

(Osamu Shimabukuro) Faculty of Education, NAgasaki University, Nagasaki, 852-8521, JAPAN

Email address: shimabukuro@nagasaki-u.ac.jp 